to really reduce down the size of the facility, and that critical work is not lost.

How will things return to normal when the time comes?

I hope that researchers should be able to return to their work as though it was the day before when they left. Obviously, that will depend on exactly how long the facility is shut down for but I anticipate we will be able to provide all of the normal services we usually do from the day the college reopens.

If we start to lose power and/ or water then stocks may have to be reduced further, and it is possible that critical lines will be lost. This may take some time to recover from-it depends how quickly we can, as a community, determine what is critical.

Interviewed by Ellen P. Neff

Published online: 15 April 2020

https://doi.org/10.1038/s41684-020-0533-1

\title{
COVID-19 Q\&A: Collaborating and coordinating keeps a zebrafish facility going
}

\author{
Christine Archer, fish facility coordinator at the University of Colorado Anschutz Medical Campus, walks Lab \\ Animal through her facility's response to the ongoing coronavirus pandemic.
}

What is your role at your facility, what animals do you care for, and what are they used for?

I am the fish facility coordinator at University of Colorado's Anschutz Medical Campus. We have a facility of approximately 25,000 zebrafish. The facility supports diverse biomedical research endeavors in neurobiology, cardiovascular and craniofacial development and more, towards a better understanding of the developmental basis of congenital defects.

How has your facility responded to COVID-19?

Our facility was instructed to shut down on March $17^{\text {th }}$. We had an idea this was coming, so a number of the labs began preparing for contingencies prior to this date. We are running the facility with our two full time animal care professionals, and for the most part the day-to-day animal care is the same as always-with a few exceptions. Only one of the two care staff come in on a day, to reduce risk to everyone. We've reduced to a maintenance feeding, where adults are fed once a day and juvies and subadults get fed twice a day. We've moved to a model where we simply spot change tanks as needed daily, instead of our regular 8-week full change schedule.

We did not have to reduce numbers, though a few labs did reduce some extra broodstock animals, to help lessen the daily check and feeding burden. A number of labs did have to stop everything with regards to embryological work they were in the

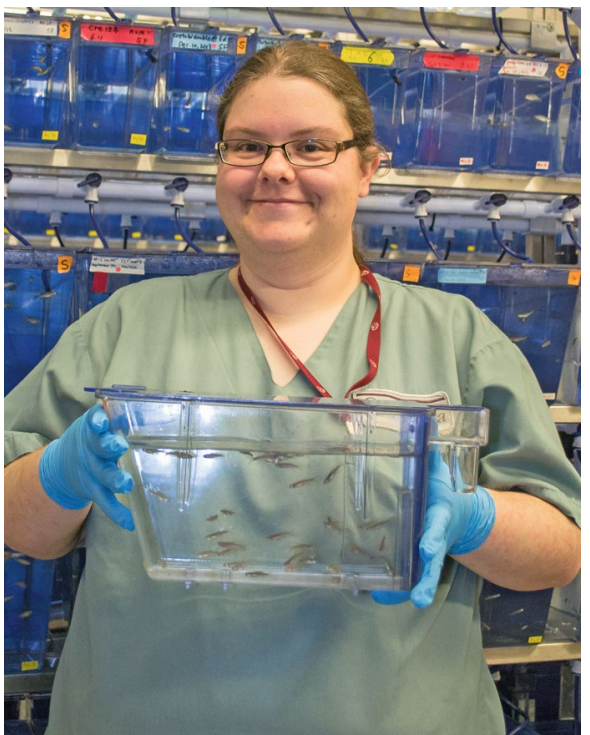

midst of, but the main housing facility was unaffected.

Every day during the shutdown, fish care folks do a morning check in via text and an afternoon debrief via email of the day's happenings and things to consider for the coming days/weeks.

While most fish users were perfectly fine with a 2-week stoppage of all animal work and zebrafish breeding, as the closure extensions have happened we've begun discussing how we should prioritize essential animals to be replaced and how we can maximize breeding to keep the fish stocks in good shape. Likely this will involve cooperation between the fish care team, who have sole access to the facility at this time, and the fish users, who are currently not permitted in the facility. We really want to limit exposure risk to the fish care team, so strict scheduling will need to occur.

\section{Did you feel prepared?}

For the most part, yes. I am actually very new to this position, having only started at the end of 2019. I feel as though I am still getting the hang of everything in this new environment with new colleagues and different ways of working with zebrafish than I am accustomed to. However, I've been working in lab animal care for more than 10 years, with most of that being zebrafish work. I'm also a bit obsessed with being very prepared for all kinds of contingencies, and I've previously written about emergency preparedness for a number of less common pet species.

The fish care team began discussing and preparing for the shutdown more than a week before it was announced. We wrote up our contingency plans as a group, collaboratively with the fish users, and got them approved by the animal care department, ensuring we had a chain of command and care prepared in the event that one or both of the animal care staff were to get sick. We worked extra hours in preparation for a potential shutdown, to make sure everything was stocked, systems were serviced, and as many tanks were changed as possible. I also wrote up very detailed pictorials that would illustrate to just about anyone how to 
check the room systems and where to find essential extra supplies.

I feel like sometimes with zebrafish, they sort of end up as an afterthought in some overall facility emergency planning. We admittedly have some knowledge gaps when it comes to best practices for their care and welfare, and frankly lots of animal care teams, through no real fault of their own, have limited husbandry knowledge for the species. These unknowns became our biggest hurdle. How little can we actually feed the fish? How often does the physical room need to be checked vs. remote monitoring? What is a viable and safe number of broodstock animals to maintain as a bare minimum? All things we are working through. I'm hoping that coming out of this there will be a renewed interest in doing really basic zebrafish husbandry studies to help determine how to better prepare for an emergency.
How will things return to normal when the time comes?

I don't anticipate there being much delay in getting things back to normal, as our current goal is to keep all existing lines going, with some essential breedings done sparingly for colony maintenance.

Thankfully when it comes to zebrafish work, the fish have most of their daily needs met with a well-managed recirculating aquaculture system overseen by competent aquarists. We may see more failed breedings and reduced fecundity for a week or two while we ramp back up to a full schedule and heavier feedings. This is something we plan to keep tabs on. For now, anecdotally the fish seem to be doing quite well with less disruption in their day!

Anything else you'd like to share? I want to thank the animal care teams everywhere for risking everything to ensure our charges get the care they need. I also want to thank, in particular, my fish users for being so accommodating in an unprecedented crisis such as this one. It has meant a lot to the fish care team that they have been so mindful of our safety during this time.

Furthermore, thank you to the Zebrafish Husbandry Association (ZHA) for being an unwavering source of insight, guidance, and support for the zebrafish husbandry community. Not just during this crisis, but all the time. Our animals and our research are better for the resources provided by ZHA.

Interviewed by Ellen P. Neff

Published online: 15 April 2020

https://doi.org/10.1038/s41684-020-0534-0 